\title{
AThrIA: a New Adaptive Threshold Identification Algorithm for Electrocardiographic P Waves
}

\author{
Agnese Sbrollini, Sofia Mercanti, Angela Agostinelli, Micaela Morettini, Francesco Di Nardo, \\ Sandro Fioretti, Laura Burattini*
}

Department of Information Engineering, Università Politecnica delle Marche, Ancona, Italy

\begin{abstract}
Proposed algorithms for $P$-wave identification and segmentation usually search for it within a window just before the $R$ peak, thus hypothesizing the presence of at most one $P$ wave, as it is in a normal electrocardiographic (ECG) tracings. In presence of abnormal atrial depolarization, however, there might be no $P$ waves (as in atrial fibrillation) or multiple $P$ waves (as in second- or third-degree atrioventricular blocks). Thus, this study proposes a new Adaptive Threshold Identification Algorithm (AThrIA) for ECG P-waves whose most innovative feature is to look for $P$ waves all along the heartbeat, potentially allowing multiple $P$ waves identification. AThrIA ability to identify and segment (finding onset, maximum and offset) $P$ waves was tested in simulated and experimental ECG tracings with no $P$ waves, one $P$ wave and two $P$ waves, respectively. All $P$ waves involved in the study were annotated. Results indicate that AThrIA correctly identified all $P$ waves (no false-negative or false-positive detections). Segmentation errors were $0 \mathrm{~ms}$ for the simulated ECG tracings, and no more than $10 \mathrm{~ms}$ for the experimental tracings. Thus, AThrIA represents a promising tool for $P$-wave identification and segmentation in both physiological (one $P$ wave) and pathological (none or multiple $P$ waves) conditions.
\end{abstract}

\section{Introduction}

The electrocardiographic (ECG) signal is the representation of electrical activity of the heart. Being noninvasive, simple and cheap, it is one of the most clinically used test for assessing cardiac abnormalities and diseases. An ECG signal is characterized by a typical morphology composed by a sequence of waveforms which are pseudo-periodically repeated, each period representing a heartbeat. Specifically, P wave represents atrial depolarization; QRS complex represents ventricular depolarization and hides atria repolarization; and $\mathrm{T}$ wave (together with $\mathrm{U}$ wave, when present) represents ventricular repolarization. Thus, abnormalities in the morphology or duration of these waves may indicate the presence of cardiac diseases.

Many segmentation algorithms have been proposed in the literature for identification of ECG waveforms. Most of them, however, focuses on QRS-complex and T-wave identification [1-4], being ventricular abnormalities more directly associated to malignant arrhythmias and sudden cardiac death [5-7]. Still, since atrial diseases like atrial fibrillation, junctional blocks and atrial tachycardia became more and more common, some algorithms have focused on P-wave identification and segmentation $[3,8$ 11] to provide information on the sinoatrial nodal activity.

$\mathrm{P}$-wave segmentation is quite challenging: $\mathrm{P}$ wave is usually $80 \mathrm{~ms}$ to $100 \mathrm{~ms}$ wide and $0.1 \mathrm{mV}$ to $0.4 \mathrm{mV}$ high [12], often represents the ECG wave with the lowest amplitude and may be strongly corrupted by noise. Usually, algorithms look for a P wave in an ECG window just before the QRS complex [8-10], thus hypothesizing the presence of at most one $\mathrm{P}$ wave, as it is in a normal ECG trace. In presence of abnormal atrial depolarization, however, there might be no $\mathrm{P}$ waves (as in atrial fibrillation) or more than one $\mathrm{P}$ waves (as in second- or third-degree atrioventricular blocks where there are two and three $\mathrm{P}$ waves, respectively). Thus, the aim of the present methodological study is to propose a new Adaptive Threshold Identification Algorithm (AThrIA) for ECG P waves, whose most innovative feature is to look for potential $\mathrm{P}$ waves all along the heartbeat.

\section{Methods}

\subsection{Adaptive Threshold Identification Algorithm for Electrocardiographic P Waves}

AThrIA looks for $\mathrm{P}$ waves all along each heartbeat, identified in a RR interval (InterRR; Fig. 1). InterRR contains several waves, including RS complex, T wave, possibly $\mathrm{U}$ wave, possibly none, one or more $\mathrm{P}$ waves (typically one), and QR complex (Fig. 2). Search for potential $\mathrm{P}$ waves occurs with an adaptive threshold (THR_Search; initially set as $0.1 \cdot \mathrm{QRS}$ amplitude) that 
determines an amplitude interval (THR_Search $\pm \delta$; $\delta=0.13 \cdot$ THR_Search) which identifies the following ECG fronts (Fig. 2): a down-going front in RS complex; an upgoing front and a down-going front in $\mathrm{T}$ wave; an horizontal front in $\mathrm{P}$ wave (which occurs when $\mathrm{P}$-wave maximum is included in THR_Search $\pm \delta$ ); and an upgoing front in QR complex. Too high values of THR_Search may lead to no T-wave front identification or to horizontal front identification in the $\mathrm{T}$ wave. In the latter case $\mathrm{T}$ wave is initially identified as a potential $\mathrm{P}$ wave, but is later rejected because too wide. When too high, THR_Search is decreased by $25 \%$ of its value until it becomes satisfactory or reaches its minimum value (after three consecutive reductions), indicating P-wave absence. When present, $\mathrm{U}$ wave is not identified as potential $\mathrm{P}$ wave because $\mathrm{U}$-wave amplitude is too low.

Potential $\mathrm{P}$ waves have horizontal fronts and undergo segmentation by identification of its maximum (Pmax; $\mathrm{ms}$ ), its onset (Pon; ms) and its offset (Poff; ms). Specifically (Fig. 2), Pmax is identified as the instant where the ECG maximum within the horizontal front occurs; Pon is the instant that maximizes the distance between ECG and the segment connecting Pmax with the ECG point located $100 \mathrm{~ms}$ before Pmax; and Poff is the instant that maximizes the distance between ECG and the segment connecting Pmax with the ECG point located 70 $\mathrm{ms}$ after Pmax. Eventually, $\mathrm{P}$ amplitude (Pamp; $\mathrm{mV}$ ) is computed as ECG amplitude in Pmax minus the minimum between ECG amplitude in Pon and ECG amplitude in Poff, and P duration (Pdur; ms) is computed as Poff minus Pon. Once a potential $\mathrm{P}$ wave has been segmented, its amplitude and its duration must both fall within predefined ranges $(\mathrm{Pamp}=0.1 \div 0.4 \mathrm{mV}$, and Pdur $=80 \div 100 \mathrm{~ms}$ ) to be confirmed and thus, identified.

\subsection{Data}

AthrIA was tested in both simulated and experimental $10 \mathrm{~s}$ ECG signals (sampling frequency: $250 \mathrm{~Hz}$ ), preprocessed using a technique based on the principal component analysis (only the first eigenvector projection was used) $[13,14]$. $R$ peaks were detected through the Pan-Tompkins algorithm [1].

Simulation study. Four simulated ECG signals (Fig. 3) were designed to test AthrIA in different controlled conditions and created using the Matlab ECG simulator (http://it.mathworks.com/matlabcentral/fileexchange/108 58-ecg-simulation-using-matlab). Each InterRR of the first one reproduced the normal PQRST wave sequence; the second one also included the U wave (PQRSTU); the third one had no P wave (QRST); and the fourth one included two $\mathrm{P}$ waves (P-PQRST). Waves amplitudes and durations were the following: $0.2 \mathrm{mV}$ and $90 \mathrm{~ms}$ for Pwaves, $-0.5 \mathrm{mV}$ and $40 \mathrm{~ms}$ for Q-waves, $3.0 \mathrm{mV}$ and 110 $\mathrm{ms}$ for $\mathrm{R}$ waves, $-1.0 \mathrm{mV}$ and $70 \mathrm{~ms}$ for S-waves, $0.4 \mathrm{mV}$ and $140 \mathrm{~ms}$ for $\mathrm{T}$-waves, and $0.05 \mathrm{mV}$ and $50 \mathrm{~ms}$ for $\mathrm{U}$

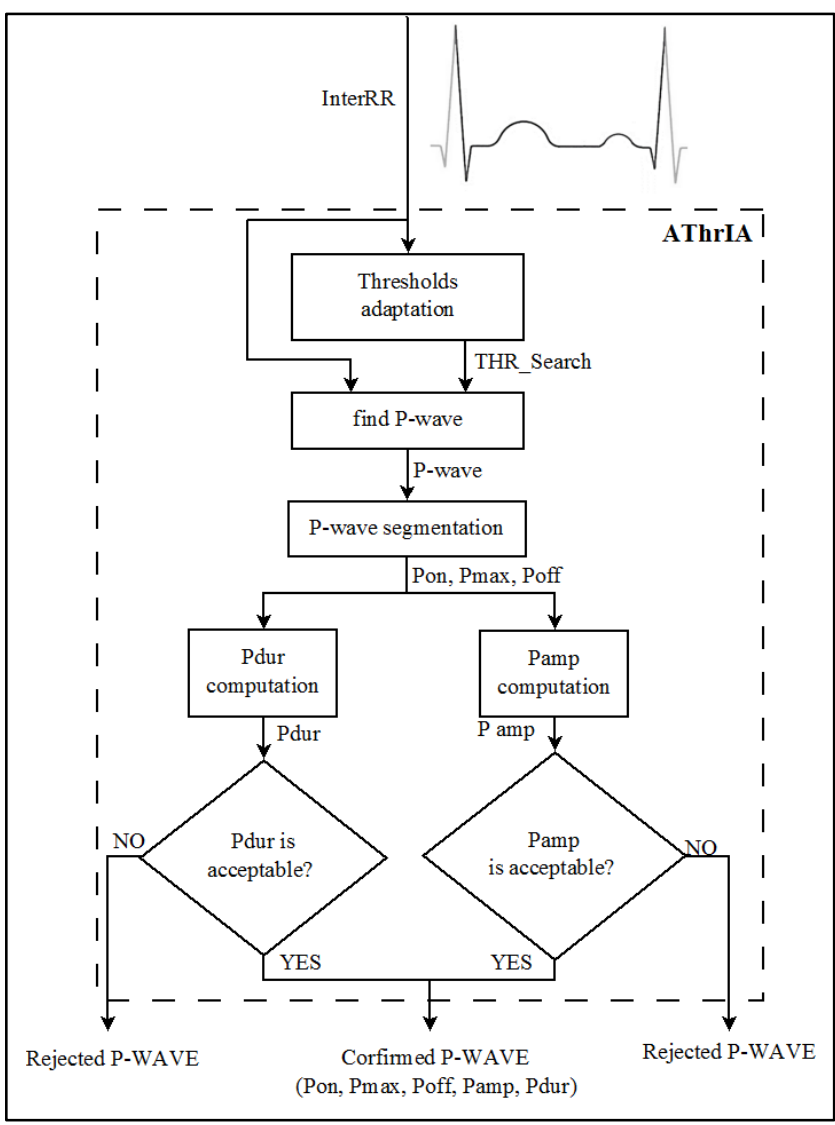

Figure1. Block diagram of AThrIA, the proposed adaptive Threshold Identification Algorithm for P-Wave Segmentation.

waves. In addition, all the simulated ECG were characterized by a heart rate (HR) of $75 \mathrm{bpm}$ and no HR variability (HRV). Occurrence of P-wave onsets, maxima and offsets were known and thus considered as annotation references.

Experimental study. Four experimental ECG signals (Fig. 3) were selected to test AthrIA in different real

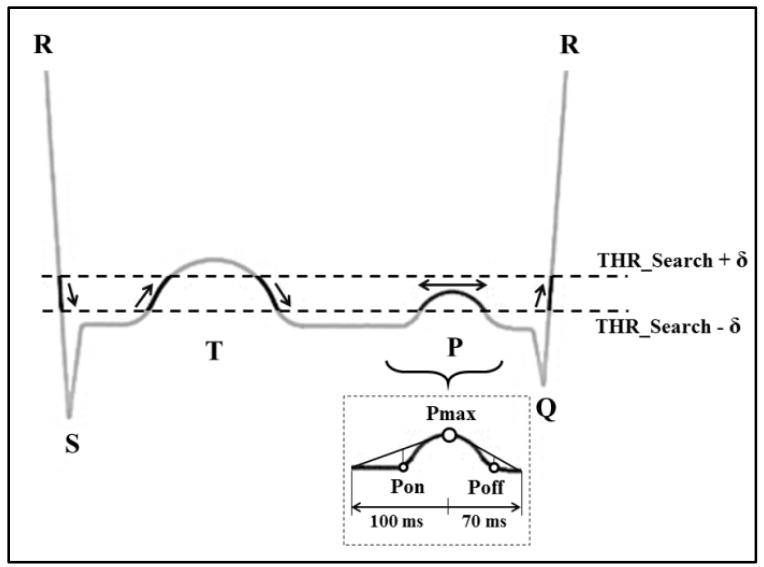

Figure 2. Potential P-wave identification by AThrIA and subsequent $\mathrm{P}$-wave segmentation. 
conditions. The first one was acquired from a normal subject and showed normal PQRST waves; the second was also acquire from a normal subject, but the tracing also showed the U wave (PQRSTU); the third one was acquired from a patient who suffered of atrial fibrillation and the ECG tracing showed no P wave; eventually, the fourth one was acquired from a patient with a seconddegree atrioventricular block so that the ECG showed two $\mathrm{P}$ waves. HR were $72 \mathrm{bpm}, 66 \mathrm{bpm}, 88 \mathrm{bpm}$ and $37 \mathrm{bpm}$, respectively; HRV were $10 \mathrm{~ms}, 48 \mathrm{~ms}, 227 \mathrm{~ms}$ and $28 \mathrm{~ms}$, respectively. P-wave onsets, maxima and offsets were visually annotated by an expert and used as references.

\subsection{Statistics}

Corresponding annotated vs AThrIA derived parameters distributions were compared by means of the T-test for equal mean. Error $(\varepsilon)$ in P-wave features computation was defined as absolute value of the difference between annotated and computed values.

\section{Results}

The results relative to AThrIA application to both simulated and experimental data are reported in Table 1.

In both studies number of annotated $P$ waves matched number of detected $\mathrm{P}$ wave (no false negative detections and no false positive detection).

No statistically significant differences were observed between mean values of corresponding annotated vs identified P-wave parameters distributions. Errors relative to the simulation study were always better than or equal to corresponding errors in the experimental study. Specifically, errors relative to the identification of Pon, Pmax and Poff were always zero for the simulation study and, on average, smaller than $10 \mathrm{~ms}$ for the experimental study. Errors relative to Pamp were zero for both simulation and clinical studies. Eventually, errors relative to Pdur were zero for the simulation study and $10 \mathrm{~ms}$ or less for the experimental study.

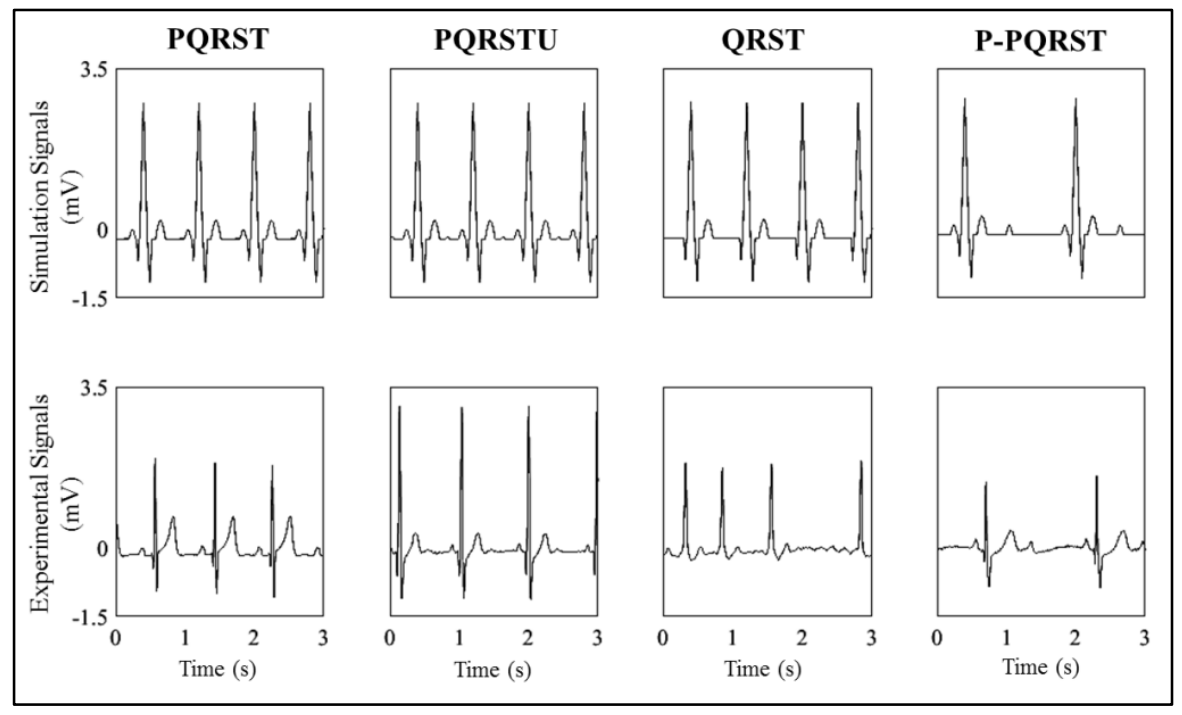

Figure 3. Three-second window of all ECG signals involved in simulation and experimental studies.

Table 1. P-wave identification in simulation and experimental studies. Errors $(\varepsilon)$ are reported as mean \pm standard deviation (n.a.: not applicable)

\begin{tabular}{ccccccccc}
\hline & & $\begin{array}{c}\text { Number of } \\
\text { annotated } \\
\text { P-wave }\end{array}$ & $\begin{array}{c}\text { Number of } \\
\text { identified } \\
\text { P-wave }\end{array}$ & $\begin{array}{c}\boldsymbol{\varepsilon} \text { Pon } \\
(\mathbf{m s})\end{array}$ & $\begin{array}{c}\boldsymbol{\varepsilon} \text { Pmax } \\
(\mathbf{m s})\end{array}$ & $\begin{array}{c}\boldsymbol{\varepsilon} \text { Poff } \\
(\mathbf{m s})\end{array}$ & $\begin{array}{c}\boldsymbol{\varepsilon} \text { Pamp } \\
(\mathbf{m V})\end{array}$ & $\begin{array}{c}\boldsymbol{\varepsilon} \text { Pdur } \\
(\mathbf{m s})\end{array}$ \\
\hline Simulated & PQRST & 11 & 11 & $0 \pm 0$ & $0 \pm 0$ & $0 \pm 0$ & $0 \pm 0$ & $0 \pm 0$ \\
ECG & PQRSTU & 11 & 11 & $0 \pm 0$ & $0 \pm 0$ & $0 \pm 0$ & $0 \pm 0$ & $0 \pm 0$ \\
Signals & QRST & 0 & 0 & n.a. & n.a. & n.a. & n.a. & n.a. \\
& P-PQRST & 10 & 10 & $0 \pm 0$ & $0 \pm 0$ & $0 \pm 0$ & $0 \pm 0$ & $0 \pm 0$ \\
\hline \multirow{2}{*}{$\begin{array}{c}\text { Experimental } \\
\text { ECG }\end{array}$} & PQRST & 10 & 10 & $3 \pm 3$ & $0 \pm 0$ & $4 \pm 2$ & $0 \pm 0$ & $3 \pm 4$ \\
Signals & PQRST & 10 & 10 & $3 \pm 3$ & $2 \pm 2$ & $3 \pm 3$ & $0 \pm 0$ & $4 \pm 4$ \\
& QRST & 0 & 0 & n.a. & n.a. & n.a. & n.a. & n.a. \\
& P-PQRT & 10 & 10 & $8 \pm 5$ & $2 \pm 3$ & $7 \pm 6$ & $0 \pm 0$ & $10 \pm 9$ \\
\hline
\end{tabular}




\section{Discussion}

This paper proposed AThrIA, a new adaptive threshold identification algorithm for ECG $\mathrm{P}$ waves. AThrIA, differently from other methods previously proposed in literature $[3,8-10]$, searches the $\mathrm{P}$ wave all along the RR interval, by allowing identification of multiple $\mathrm{P}$ waves, when present. In this study, the method was tested in both simulated and experimental ECG tracings. Results indicate that P-wave identification occurred with no errors (no false-positive or false-negative detections). Segmentation points (Pon, Pmax and Poff) were compared to annotated values. In the simulation study annotations were set in the points where the waveforms used to model the $\mathrm{P}$ waves are connected to the baseline. Such connections perfectly determine the beginning and the end of the $\mathrm{P}$ waves but differ from the real cases in which transitions from baseline to $P$ waves and vice versa occur gradually. In the experimental ECG tracings annotations were visually set by a human expert who may hardy distinguish points represented by two consecutive samples (4 ms). Despite the limitations in the annotations, AThrIA provided segmentation points identification with errors usually lower than $10 \mathrm{~ms}$. Such precision is of the order of magnitude used for segmentation of other waves (for example T-wave offset) [4]. Thus, according to the present results, AThrIA appears as a promising tool for Pwave segmentation and consequent atrial diseases classification (like atrial fibrillation, junctional blocks or atrial tachycardia) based on number of identified $P$ waves in each heartbeat. Future studies on larger datasets characterized by different levels of noise and representing different ECG atrial diseases are needed to confirm the goodness of the preliminary results reported in the present methodological study.

\section{Conclusion}

AThrIA represents a promising tool for P-wave identification and segmentation in both physiological (one $\mathrm{P}$ wave) and pathological (none or multiple $\mathrm{P}$ waves) conditions.

\section{References}

[1] Pan J, Tompkins WJ. A real-time QRS detection algorithm. IEEE Transactions on Biomedical Engineering 1985; 32:230-6. Doi:10.1109/TBME.1985.325532.

[2] Laguna P, Thakor NV. New algorithm for QT interval analysis in 24-hour Holter ECG: performance and application. Medical \& Biological Engineering \& Computing 1990; 28:67-73.

[3] Martínez A, Alcaraz R, Rieta JJ. Application of the phasor transform for automatic delineation of single-lead ECG fiducial points. Physiological Measurement 2010; 31:146785. Doi:10.1088/0967-3334/31/11/005.

[4] Agostinelli A, Giuliani C, Burattini L. Use of the dominant $\mathrm{T}$ wave to enhance reliability of $\mathrm{T}$-wave offset identification. Journal of Electrocardiology 2014; 47:98105. Doi:10.1016/j.jelectrocard.2013.09.007.

[5] Man SC, De Winter PV, Maan AC, Thijssen J, Borleffs JW, van Meerwijk WP, Bootsma M, van Erven L, van der Wall EE, Schalij MJ, Burattini L, Burattini R, Swenne CA. Predictive Power of T-wave Alternans and of Ventricular Gradient 2 Hysteresis for the Occurrence of Ventricular Arrhythmias in Primary Prevention ICD Patients. Journal of Electrocardioliology 2011; 44:453-9. Doi:10.1016/j.jelectrocard.2011.05.004.

[6] Burattini L, Zareba W, Burattini R. Identification of gender-related normality regions for $\mathrm{T}$-wave alternans. Annals of Noninvasive Electrocardiology 2010; 15:328-36. Doi: 10.1111/j.1542-474X.2010.00388.x.

[7] Zareba W, Cygankiewicz I. Long QT syndrome and short QT syndrome. Progress in Cardiovascular Diseases 2008; 51:264-78. Doi: 10.1016/j.pcad.2008.10.006.

[8] Pürerfellner H, Pokushalov E, Sarkar S, Koehler J, Zhou R, Urban L, Hindricks G. P-wave evidence as a method for improving algorithm to detect atrial fibrillation in insertable cardiac monitors. Heart Rhythm 2014; 11: 1575-83. Doi: 10.1016/j.hrthm.2014.06.006.

[9] Saini I, Singh D, Khosla A. K-nearest neighbour-based algorithm for P- and T-waves detection and delineation Journal of Medical Engineering \& Technology 2014; 38:115-24. Doi: 10.3109/03091902.2014.882424.

[10] Lin B, Wang PJ, Mahapatra S, Homoud M, Link M, Estes NA, Al-Ahmad A. Extraction of buried $\mathrm{P}$ waves from printed electrocardiograms. Annals of Noninvasive Electrocardiology 2005; 10:142-5. Doi: 10.1111/j.1542474X.2005.05605.x.

[11] Kennedy A, Finlay DD, Guldenring D, Bond RR, McLaughlin J. Detecting the elusive P-wave: a new ECG lead to improve the recording of atrial activity. IEEE Transactions on Biomedical Engineering 2016; 63:243-9. Doi: 10.1109/TBME.2015.2450212.

[12] Klabunde RE. Cardiovascular Physiology Concepts. Wolters Kluwer/Lippincott, Williams \& Wilkins, 2011, 2nd Edition.

[13] Mourad T. ECG Denoising based on PCA and using R Peaks detection. In Proceedings of the International Conference on Bio-inspired Systems and Signal Processing, Lisbon, Portugal, BIOSIGNALS, 2015; 1:119-125.

[14] Romero I. PCA and ICA applied to noise reduction in multi-lead ECG. Computing in Cardiology 2011; 38:613-6.

Address for correspondence.

Laura Burattini.

Department of Information Engineering,

Università Politecnica delle Marche,

via Brecce Bianche 12,

60131, Ancona, Italy.

E-mail address. 1.burattini@univpm.it. 